\title{
Probabilistic instantaneous quantum computation
}

\author{
Časlav Brukner ${ }^{1}$, Jian-Wei Pan $^{1}$, Christoph Simon ${ }^{2}$, Gregor Weihs ${ }^{1}$, and Anton Zeilinger ${ }^{1}$ \\ ${ }^{1}$ Institut für Experimentalphysik, Universität Wien, Boltzmanngasse 5, A-1090 Wien, Austria \\ ${ }^{2}$ Centre for Quantum Computation, University of Oxford, \\ Parks Road, Oxford OX1 3PU, United Kingdom
}

(Dated: December 3, 2018)

\begin{abstract}
The principle of teleportation can be used to perform a quantum computation even before its quantum input is defined. The basic idea is to perform the quantum computation at some earlier time with qubits which are part of an entangled state. At a later time a generalized Bell state measurement is performed jointly on the then defined actual input qubits and the rest of the entangled state. This projects the output state onto the correct one with a certain exponentially small probability. The sufficient conditions are found under which the scheme is of benefit.
\end{abstract}

PACS numbers: 03.67.-a, 03.65.-w, 03.67.-Hk, 03.67.Lx

Quantum computers 1 - a new type of machine that exploits the quantum properties of information - could perform certain types of calculations with speedup over any foreseeable classical computer. Quantum teleportation 2] - one of the most basic information procedures in quantum mechanics - enables transmission and reconstruction of a general quantum state over arbitrary distances. In 1997 Nielsen and Chuang [3] have shown that the principle of teleportation can be used to perform universal quantum computation in a probabilistic fashion. Since then a substantial amount of work has been done on generalizations and applications of the idea. Gottesman and Chuang [4] developed fault-tolerant constructions of quantum gates. Knill et al. [5] apply the scheme to propose quantum computation with linear optics together with single-photon detection and single-photon sources. Further extensions have been developed [6, 7, 8, 9, 10].

Here we consider the time aspect of teleportation-based quantum computation. We show that with finite probability the computational time of an arbitrary long quantum computation can be saved completely. The basic idea is to use quantum teleportation to perform the entire quantum computation even before its quantum input is defined. This then allows with certain exponentially small probability to obtain the output of the computation immediately after its input is given. The sufficient conditions are found under which the scheme is of benefit.

Imagine that an engineer is given a certain problem of such a complexity that in order to obtain its solution within reasonable time she has to use a quantum computer. Suppose that the conditions on the quantum computation are the following:

1. At time $t_{1}$ the engineer is given an input to the quantum computation in an arbitrary quantum state unknown to her.

2. The engineer is required to give the output of her computation at time $t_{2}$. If however she is not sure that her output is the right one (e.g. because her computer has not finished the computation before $\left.t_{2}\right)$ she is allowed not to give any state. Such a situation is denoted by "no answer".
3. The engineer is strongly advised not to overestimate her computational resources. By this we mean that the engineer's choice to give no answer is not evaluated negatively, and that an incorrect result is evaluated more negatively than the correct one is evaluated positively (e.g. one may imagine that she obtains $\mathrm{P}$ positive points for the correct result, 0 points for no answer, and $\mathrm{N}$ negative points for an incorrect result, where $\mathrm{N}$ is much larger than P.).

For the purpose of evaluating the engineer's computation, one may imagine that whenever the engineer decides to give the output of her computation it is subjected to a kind of "check-measurement" in a basis in which one of the basis states is the right output state. Denote the outcome corresponding to the right output by $O$. Then, only if the measurement gives the result $O$ does the engineer gain $\mathrm{P}$ points, otherwise she loses $\mathrm{N}$ points.

What are useful typical situations where the input of the computation is an arbitrarily and unknown quantum state as required in 1? All known quantum computation algorithms start with classical inputs somewhere along the line. To our knowledge only the quantum simulations 11] and certain quantum cryptography protocols start with quantum inputs. Also realistic situations where the duration of a quantum computation is restricted by a deadline are not typical. Is there any example for the situations described by points 1.-3? We give an explicit example for such situation for quantum simulations later in the text. Here we note that the input in universal quantum operations, like universal quantum cloning 12 or universal quantum universal-NOT operation [13] is an arbitrary and unknown quantum state 14].

We now consider possible strategies which the engineer can follow. Normally, after the engineer gets the input $n$ qubits (qubits 1 ) for the quantum computation, she feeds them into her computer and starts the quantum computation. Now assume the quantum computation is very time-consuming, so that this procedure is not fast enough and the quantum computer does not terminate before the deadline at $t_{2}$, as illustrated in Fig. Th. In such a situation the engineer, for instance, can decide not to 


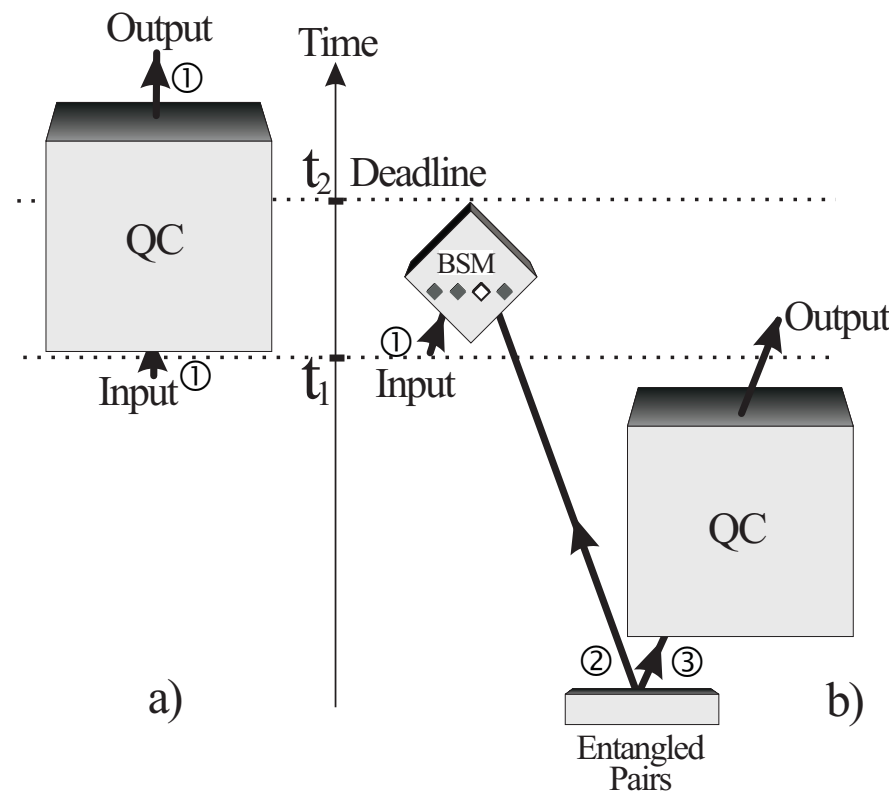

FIG. 1: a) Conventional scheme: At time $t_{1}$ the engineer is given the input qubits 1 of the quantum computation (QC) in a quantum state unknown to her. She feeds them into her quantum computer and starts the computation. The computation is very time-consuming, so that the quantum computer does not terminate before the deadline at $t_{2}$. b) Scheme for instantaneous quantum computation: At a time earlier than $t_{1}$ the engineer has fed qubits 3 , which are each maximally entangled with one qubit 2 , into her quantum computer and has done the computation. At the later time $t_{1}$ when the input qubits 1 are given to her the engineer performs a Bellstate measurement (BSM) on each pair of qubits 1 and 2 and projects qubits 3 onto a corresponding state. In a certain exponentially small fraction of cases the computational time is saved completely as she immediately knows that qubits 3 are projected onto the output state resulting from the correct input one.

give an answer, which results in a total of zero points. Alternatively, she can choose any state at random and give this as the output of her computation. This however leads to a high negative score because the probability of $1-(1 / 2)^{n}$ not to obtain the result $O$ in the checkmeasurement is higher than the one of $1 / 2^{n}$ to obtain this result for a n-qubit state $(n>1)$ chosen at random.

We will now show that there is an alternative strategy where the engineer can have a positive score. There she can obtain the exact output state of an arbitrarily long quantum computation instantaneously with some probability. Most importantly, she knows when she obtains the correct output state and thus can pass it on for evaluation. The strategy uses teleportation-based quantum computation [3].

Quantum teleportation [2] is the transmission and reconstruction over arbitrary distances of the state of a quantum system. During teleportation, an initial system in the state that is to be transferred and one of a pair of entangled subsystems are subjected to a Bell state measurement, such that the second subsystem of the entangled pair acquires the state of the initial system. The later subsystem is brought into the state of initial system by an accordingly chosen transformation after receiving via classical communication channel the information which of the Bell-state results was obtained.

Now imagine that the engineer has $n$ entangled pairs of qubits 2 and 3 . She can feed members of the entangled pairs (qubits 3) into her quantum computer long before $t_{1}$, when the actual input for her computation is given to her, as illustrated in Fig. 1 $1 \mathrm{~b}$. This means that during the computation the qubits 3 in her quantum computer are entangled to the qubits 2 . At some point her computation will terminate and output qubits 3 .

As soon as she obtains the input qubits 1 the engineer performs the Bell state measurement on each of $n$ pairs of qubits 1 and 2 . In $(1 / 4)^{n}$ cases the whole state of qubits 3 is projected onto the state resulting from the correct input and she does not have to perform any additional transformation on qubits 3 . In the remaining $1-(1 / 4)^{n}$ cases, the result of the engineer's Bell state analysis will not be the right one. However, in a situation (as defined by conditions 1-3 given above) where it is of advantage to both have the correct output state for a problem and to know it at a very early time even if only with small probability, our scheme is of benefit.

We now consider also the remaining cases. In the usual teleportation procedure, the engineer would have to perform single-qubit unitary transformation on her qubits 3 . But now she has already fed them into her quantum computer. Because the set of unitary transformations necessary to complete the usual teleportation procedure and her computation do not always commute, in general she has to invert the full quantum computation performed so far, perform the single-qubit transformations required by the teleportation procedure, and start the quantum computation again [15]. The scheme is thus only efficient for the case where no transformation are done after the Bell-state measurement. This results in a total computational time twice as long as the time needed for the conventional computation (if we neglect the time required for the single-qubit transformations). This means that in a fraction $1-(1 / 4)^{n}$ of all cases in general our scheme will not help the engineer to meet her deadline. In those cases the best strategy for her is to give no answer.

But in the succesful case, depending on the length of her computation, the scheme for instantaneous computation may constitute an enormous gain in time, which might be decisive in situations as specified by conditions 1-3 listed above. Following the evaluation criterion 3 , the averaged point score gained in our scheme is $S_{\text {inst }}=P(1 / 4)^{n}$, which exceeds both the score of $S_{n o}=0$ if the engineer constantly provides no answer, and the score of $S_{\text {rand }}=P\left(1 / 2^{n}\right)-N\left(1-1 / 2^{n}\right)$, if she constantly chooses the output at random.

It is important to note that in our protocol the resources are consumed even in the cases when the engineer does not provide an answer. The reason is that the en- 
gineer's computation has to be run each time thus using up $n$ entangled pairs. Moreover, since the probability of success is $(1 / 4)^{n}$, there is an expected exponential cost of entangled pairs to get a single successful run of the computation. If, for example, each computation requires one hour, but $n_{0}=1000$ attempts must be made (which is the approximate number $4^{n}$ for $n=5$ ), the company that distributes entangled pairs has to be paid for 1000 hours, not just one hour. There are however situations where it clearly still pays off for the engineer to follow our scheme. These are when the engineer's gain $\mathrm{P}$ (which in our example could correspond to a certain amount of money) is larger than the overall costs incurred over the average number of attempts required to get one successful run, i.e. when $P>n_{0} C$, where $C$ are the costs of a single run.

It should be stressed again that the protocol based on teleportation is only reasonable to consider if the engineer's input is in an arbitrary quantum state unknown to her. In contrast, if the input is a classical one, then a simple protocol is possible which even do not require entanglement but has a success rate higher than the teleportation-based one. Suppose that the input still is in the state unknown to the engineer, but this state is one of $2^{n}$ (orthogonal) states which are known in advance to the engineer. Then the engineer simply can perform the computation in advance choosing any of the $2^{n}$ states as the input. Later, when the actual input is given to her, she can measure it in the basis build out of the $2^{n}$ states. On the basis of the result obtained she then knows with certainty whether she had performed the computation with the correct input or not. With probability $1 / 2^{n}$ her input will be the correct one, when she passes on her output; otherwise she provides no answer.

Another situation where requirement 1 is not fulfilled is when the input is in an arbitrary quantum state, but this state becomes known to the engineer at the time the input is given to her. In such a situation a protocol is possible which does require entanglement but not Bellstate measurement and has a success rate higher than the teleportation-based one. The protocol is based on the "remote state preparation" [16]. The engineer starts with $n$ pairs of entangled qubits 2 and 3 and processes the computation on qubits 3 in advance. When she learns what is the actual input state, she performs such a measurement on qubits 2 to project qubits 3 onto the correct output state. The probability of success for the scheme is $1 / 2^{n}$.

There are also alternative schemes [17] which fulfill requirement 1 but give an output state only very close to the correct one and, thus, still with a finite probability to show the wrong result when measured in the checkmeasurement. In contrast with our scheme, there the engineer cannot infer with certainty whether her output state is the correct one or not. Therefore if she decides to pass on her output state, there will always be a certain probability not to obtain the result $O$ in the checkmeasurement, which consequently leads to a negative av- erage point score. Clearly such a scheme can never be better than ours, for penalty $\mathrm{N}$ for a wrong result sufficiently larger than gain $\mathrm{P}$ for a correct one.

The teleportation-based protocol scheme can also be applied in cases where, for some reason, parts of a quantum computation are performed at distant locations [8, 18]. Imagine two people, Alice and Bob, in two distant locations, each of them performing part of a common quantum computation under conditions 1-3. Suppose that the output qubits (identified with qubits 1 in our scheme) of Alice's quantum computer are an essential input for Bob's computation. Suppose also that Bob's part of computation is very time-consuming.

Imagine that entangled pairs of qubits (identified with qubits 2 and 3 in our scheme) are distributed to Alice and Bob over some quantum network in advance. Bob now can immediately feed his members of the entangled pairs (qubits 3) into his quantum computer. Thus, he can start his time-consuming computation long before the input of Alice's part of the computation is given at $t_{1}$. At some point after $t_{1}$ Alice's computation will terminate and output the qubits 1 that Bob needs. They can now proceed as in the usual quantum teleportation procedure. Alice performs the Bell state measurement on each of the pairs qubits 1 and 2. Because Bob has been able to start his computation much earlier than Alice, it is natural to assume that Bob's computation has terminated by the time Alice's call reaches him. Note that even if Alice had not done her computation before Bob did, or even started, his computation, if separation between them is large enough, he has enough time to do his calculation before Alice's call arrives. They proceed now analogous to the scheme given in the text above. If Alice tells him that the result of her Bell state measurement was the good one, he immediately knows that the output of his quantum computer is the correct one. Only in those cases he passes on the output of his computation, otherwise he provides no answer. This again results in averaged point score of $S_{\text {inst }}$ as given above.

We now give an explicit example for a situation described by conditions 1.-3. Although it admittedly might appear to be contrived to some extent, we are convienced that the existence of even just one example points at novel conceptual possibilities. The example is based on quantum simulations 11]. Imagine that our engineer is still a student of engineering. Suppose that she has a series of exams which each consists of performing a specific quantum simulation. Which ones is known to her in advance (e.g. the particular "exam question" is given to students a day before the exam takes place). Further, suppose that the process of the examination and the evaluation of its success is executed according to the rules 1-3. The reason that the input is in an arbitrary quantum state unknown to students is to prevent them to find out the correct solution just on the basis of the known solutions from the previous generations of students. Now assume that in order to pass the exams students need to have a positive score of points averaged 
over the series. The points are given on the basis of the check-measurement performed by an examiner who knows the correct output state and thus can choose the adequate measurement basis. In order to ascertain the best of them the examiner faces the students with seemingly unsolvable problem of performing a series of quantum simulations which each consume more time than the duration of a single exam. Obviously only if our student applies the teleportation-based scheme she can expect to have the correct result in a certain fraction of the total number of the exams and thus having a positive score of points to pass the exams.

In summary, we have shown that, using the principle of teleportation, one may achieve instantaneous quantum computation where: (a) one can obtain the output of an arbitrarily long computation with non-zero probability in zero computational time, i.e. immediately after its quantum input is defined, and (b) one knows when the output is correct without knowing the output. We identify the sufficient conditions under which the scheme is more efficient than any alternative one. We suggest that the ability to perform a quantum computation even before its input is defined derives from the possibility to process information represented by a quantum state without need of reading the state beforehand.

We would like to thank Terry Rudolph and Tomas Tyc for helpful comments and discussions. This work has been supported by the Austrian Science Foundation FWF, Project No. F1506 and by the QIPC Program of the European Union.
[1] M. A. Nielsen and I. L. Chuang, Quantum Computation and Quantum Information, Cambridge University Press, 2000, UK.

[2] C. H. Bennett, G. Brassard, C. Crépeau, R. Jozsa, A. Peres, and W. K. Wootters, Phys. Rev. Lett. 70, 1895 (1993).

[3] M. A. Nielsen and I. L. Chuang, Phys. Rev. Lett. 79, 321-324 (1997).

[4] D. Gottesman and I. L. Chuang, Nature 402, 390-392 (1999).

[5] E. Knill, R. Laflamme, and G. J. Milburn, Nature 409, 46 (2001).

[6] W. Dür and J. I. Cirac, Phys, Rev. A 64, 012317.

[7] G. Vidal and J. I. Cirac, preprint e-print quant-ph/0012067 at (xxx.lanl.gov); G. Vidal, L. Masanes, and J. I. Cirac, preprint quant-ph/0102037 at (xxx.lanl.gov)

[8] S. F. Huelga, J. A. Vaccaro, and A. Chefles, Phys. Rev A, 63, 042303.

[9] M. A. Nielsen, preprint quant-ph/0108020 at (xxx.lanl.gov) and references therein.

[10] M. Hillery, V. Buzek, and Mário Ziman, Phys. Rev. A 65, 022301 (2002).

[11] R. P. Feynman, Int. J. Theo. Phys. 21, 467 (1982); S. Lloyd, Science 273, 1073 (1996).

[12] V. Buzek and M. Hillary, Phys. Rev. A 54, 1844 (1996).

[13] V. Bužek, M. Hillery, R. F. Werner, Phys. Rev. A 60, R2626 (1999).

[14] Note that situation of an eavesdropper in certain quantum cryptography protocols might be similar to the situation of our engineer. For example, the input for eavesdropper operations is usually a quantum state unknown to her. Also one can assume that her operations need to be performed in a restricted time, for example, for the reason not to be detected by the parties following the protocol by noticing a delay in the exchange of the quantum states between the parties, which could be caused by the eavesdropper's action. Of course this has nothing to do with security based on detecting the state disturbance produced by an eavesdropping strategy.

[15] In certain specific cases the resulting composite transformation could be simpler to perform than the three trans- formations successively. Quantum gates can be divided into different families $C_{k}$ (see Ref. [4]). The class $C_{1}$ represents the Pauli group (Pauli operators), $C_{2}$ represents the Clifford group (including C-NOT gate, Hadamard gate, $\pi / 2$-shifts), $C_{3}$ contains the Toffoli gate etc. The classes of gates are defined by $C_{k} \equiv\left\{U \mid U C_{1} U^{\dagger} \subseteq C_{k-1}\right\}$. It is assumed that the gates in the class $C_{k}$ are more difficult to perform than the ones in $C_{k-1}$. This implies that if our quantum computation $U$ belongs to the group $C_{k}$ of gates, then the composite transformation of first undoing the computation, single qubit transformations (Pauli operators), and redoing the computation is equivalent to the simpler transformation from the class $C_{k-1}$. In particular, if $U$ belongs to $C_{2}$, then the composite transformation is a simple single-qubit rotation. The gates belonging to any specific $C_{k}$ are not sufficient to perform a universal quantum computation as this requires performance of arbitrary single-qubit transformation together with the C-NOT gate. However, as noted in Ref. 4] the set of gates of the Clifford group play an important role in the theory of quantum error-correcting codes and fault-tolerance (see D. Gottesman, Phys. Rev. A 57, $127(1998))$ and the $\pi / 2^{k}$ rotations appear in the Shor algorithm.

[16] C. H. Bennett, D. P. DiVincenzo, and P. W. Shor, Phys. Rev. Lett. 87, 077902 (2001); I. Devetak and T. Berger, Phys. Rev. Lett. 87, 197901 (2001); A. K. Pati, quant-ph/9907022 H.-K. Lo, quant-ph/9912009

[17] We give an example for the alternative scheme for the case of a one-qubit computation. At a time earlier than $t_{1}$ the engineer has taken any state, say state $|0\rangle$, as an input of her computation and has performed the computation on it. At the later time $t_{1}$ when the actual input is given to her, she measures it in the basis $\{|0\rangle,|1\rangle\}$. If the result is $|0\rangle$, she passes on the output state of her computation and if the result is $|1\rangle$, she passes on the state which is obtained by applying the optimal universal NOT [13] on the output. The engineer uses the optimal universal NOT because she does not know the output state of her computation.

[18] A. Zeilinger, Sci. Am. 32 (April 2000). 